\title{
CENTRAL MULTIPLIER THEOREMS FOR COMPACT LIE GROUPS
}

\author{
BY RONALD R. COIFMAN AND GUIDO WEISS ${ }^{1}$
}

Communicated by Elias Stein, June 22, 1973

The purpose of this note is to describe how central multiplier theorems for compact Lie groups can be reduced to corresponding results on a maximal torus. We shall show that every multiplier theorem for multiple Fourier series gives rise to a corresponding theorem for such groups and, also, for expansions in terms of special functions.

We use the notation and terminology of N. J. Weiss [4]. Let $G$ denote a simply connected semisimple Lie group, $\mathfrak{g}$ its Lie algebra and $\mathfrak{h}$ a maximal abelian subalgebra; $P^{+}$the set of positive roots in $\mathfrak{h}^{*}$, the dual of $\mathfrak{h}$ (with respect to some order), and $($,$) is the inner product on \mathfrak{h}^{*}$ induced by the Killing form. With $\lambda=\left(\lambda_{1}, \cdots, \lambda_{l}\right) \in \mathbf{Z}^{l}$ we associate the weight $\lambda=\sum_{i=1}^{l} \lambda_{i} \pi_{i}$, where $\pi_{i}$ are the fundamental weights adapted to the simple roots. The characters $\chi_{\lambda}$ of $G$ are then indexed by those $\lambda$ with nonnegative integer coefficients. The degree $d_{\lambda}$ of the corresponding representation is then given by

$$
d_{\lambda}=\prod_{\alpha \in P^{+}}(\lambda+\beta, \alpha) / \prod_{\alpha \in P^{+}}(\beta, \alpha)
$$

where $\beta=\frac{1}{2} \sum_{\alpha \in P+} \alpha$. We now define the difference operator $\mathscr{D}$ on sequences $m_{\lambda}, \lambda \in \mathbf{Z}^{l}$, by first putting $D_{\alpha} m_{\lambda}=m_{\lambda-\alpha}-m_{\lambda}$ (where the root $\alpha$ is identified with its coordinates with respect to the basis of $\pi_{i}$ 's) and then letting

$$
\mathscr{D} m_{\lambda}=\left(\prod_{\alpha \in P^{+}} D_{\alpha}\right) m_{\lambda}
$$

this is a difference operator of order $(n-l) / 2(n=\operatorname{dim} G, l=\operatorname{dim} \mathfrak{h})$.

A central convolution operator $M$ on $G$ admits a formal expansion $M \sim \sum_{\lambda_{i} \geqq 0} d_{\lambda} m_{\lambda} \chi_{\lambda}$. The sequence $\left\{m_{\lambda}\right\}$ is called a multiplier for $L^{p}(G)$ if the operator $M * f=\sum d_{\lambda} m_{\lambda}\left(\chi_{\lambda} * f\right)$, defined for generalized trigonometric polynomials $f$ (see [3]), can be extended to a bounded operator on $L^{p}(G)$.

AMS (MOS) subject classifications (1970). Primary 43A75, 42 A18.

Key words and phrases. Multipliers, maximal torus, compact Lie groups.

${ }^{1}$ This research was supported, in part, by U.S. Army Grant \#DA-ARO-D-31-12472-G143. 
The symmetric trigonometric polynomials on $\mathfrak{h} / \mathbf{Z}^{l}$ are defined by $C_{\lambda}(\tau)=$ $\sum_{\sigma \in \omega} e^{i(\lambda, \sigma(\tau))}$, where $W$ is the Weyl group. We can now state

THEOREM I. $\left\{m_{\lambda}\right\}$ defines a bounded operator on $L^{p}(G), 1 \leqq p \leqq \infty$, if $\sum_{\lambda_{i} \geqq 0} \mathscr{D}\left(d_{\lambda} m_{\lambda}\right) C_{\lambda}(\tau)$ defines a bounded operator on $L^{p}\left(\mathfrak{h} / \mathbf{Z}^{l}\right)$. (If one coordinate $\lambda_{i}$ is negative, $m_{\lambda}=0$.) In addition, for $p=1$ the condition is necessary and sufficient. For $1<p<\infty$ it is enough to assume that $\sum \mathscr{D}\left(d_{\lambda} m_{\lambda}\right) e^{i(\lambda, \tau)}$ defines a bounded operator on the torus.

We obtain the result of N. J. Weiss on $L^{p}(G)$ by using Hörmander's multiplier theorem for the torus $T^{l}$. The estimates on $\mathscr{D}\left(d_{\lambda} m_{\lambda}\right)$ can be obtained by observing that $\mathscr{D}$ is a difference operator of order $(n-l) / 2$ and $d_{\lambda}$ is a polynomial in $\lambda$ of degree $(n-l) / 2$ satisfying the estimate $\left|d_{\lambda}\right| \leqq C|\lambda|^{(n-l) / 2-1}$ on the walls of the Weyl chamber (see N. J. Weiss [4]).

We would like now to illustrate this result in the case of $S U(2)$ for which we use the notation of Coifman and Weiss [3]. The irreducible representations are indexed by the half integers $\lambda=0, \frac{1}{2}, 1, \frac{3}{2}, \cdots, d_{\lambda}=(2 \lambda+1)$ and $\chi_{\lambda}(e(\theta))=\sin (2 \lambda+1) \theta / \sin \theta$, where

$$
e(\theta)=\left(\begin{array}{cc}
e^{i \theta} & 0 \\
0 & e^{-i \theta}
\end{array}\right) .
$$

In this case $\mathscr{D} m_{\lambda}=D_{\alpha} m_{\lambda}=m_{\lambda}-m_{\lambda-1}$. The theorem now reads as follows:

The sequence $\left\{m_{\lambda}\right\}$ is a multiplier for $L^{p}(S U(2))$ if the series

$$
\sum \mathscr{D}\left[(2 \lambda+1) m_{\lambda}\right] \cos (2 \lambda+1) \theta
$$

defines a bounded operator on $L^{p}$ of the circle or, equivalently, $(2 \lambda+1) m_{\lambda}-$ $(2 \lambda-1) m_{\lambda-1}=\mu_{2 \lambda}$ is a multiplier for Fourier series.

As a consequence we obtain, by identifying special functions on $S U(2)$ as Jacobi polynomials $P_{k}^{\alpha, \beta}$, with $\alpha, \beta$ integers :

COROLlaRY. The operator $M$ which assigns to the expansion

$$
f(x)=\sum_{0}^{\infty} \alpha_{k} P_{k}^{(\alpha, \beta)}(x)(1-x)^{\alpha / 2}(1+x)^{\beta / 2}
$$

the expansion

$$
M(f)(x)=\sum_{0}^{\infty} m_{k} \alpha_{k} P_{k}^{(\alpha, \beta)}(x)(1-x)^{\alpha / 2}(1+x)^{\beta / 2}
$$

is bounded on $L^{p}([-1,1], d x)$ if the sequence $(k+1) m_{k}-(k-1) m_{k-1}$ defines an even $L^{p}$ multiplier for Fourier series.

Another easy consequence is a theorem of Bonami and Clerc [1] stating that $\left\{m_{\lambda}\right\}$ is a multiplier on $S U(2)$ if $\sum_{2^{N}}^{2^{N+1}} \lambda\left|m_{\lambda+1}-2 m_{\lambda}+m_{\lambda-1}\right| \leqq C$. This 
simply follows from our result and the theorem of Marcinkiewicz on the circle.

The proof of our theorem involves two steps. First we pass from a multiplier on $G$ to a multiplier on $T^{l}$ by means of an identity involving the Weyl character formula. Then the desired $L^{p}$ inequalities are obtained by transferring inequalities on $T^{l}$ to $G$ as is done in [2]. A similar method is valid for symmetric spaces (not necessarily of compact type) reducing the study of spherical convolution operators to the study of associated operators on the group $A$ appearing in an Iwasawa decomposition $G=$ $K A N$. This will be done in a forthcoming paper.

\section{REFERENCES}

1. Aline Bonami and Jean-Louis Clerc, Sommes de Cesàro et multiplicateurs des developpements en harmoniques spheriques, Trans. Amer. Math. Soc. (to appear).

2. Ronald R. Coifman and Guido Weiss, Operators transferred by representations of an amenable group, Proceedings of the AMS 1972 Summer Institute on Harmonic Analysis on Homogeneous Spaces, Williamstown, Mass.

3. - Analyse harmonique noncommutative sur certains espaces homogenes, Lecture Notes in Math., vol. 242, Springer-Verlag, Berlin, 1971.

4. N. J. Weiss, $L^{p}$ estimates for bi-invariant operators on compact Lie groups, Amer. J. Math. 94, (1972), 103-118. MR 45 \#5278.

Department of Mathematics, Washington University, St. Louis, MisSouri 63130 